\title{
Análise epidemiológica da hanseníase na Microrregião de Tucuruí, Amazônia brasileira, com alto percentual de incapacidade física e de casos entre jovens
}

\section{Epidemiological analysis of leprosy in Tucuruí Microregion, Brazilian Amazon with high percentage of physical disability and cases among young people}

\author{
Leandro Araújo Costa', Cláudio Joaquim Borba-Pinheiro ${ }^{2,3}$, Juliana Henrique dos Reis ${ }^{4}$, Sílvio Henrique dos Reis Júnior ${ }^{3}$ \\ ' Universidade Federal do Pará, Belém, Pará, Brasil \\ 2 Instituto Federal de Educação, Ciência e Tecnologia do Pará, Tucuruí, Pará, Brasil \\ ${ }^{3}$ Universidade do Estado do Pará, Tucuruí, Pará, Brasil \\ ${ }^{4}$ Centro Universitário Estácio do Ceará, Fortaleza, Ceará, Brasil
}

\begin{abstract}
RESUMO
OBJETIVO: Verificar o perfil epidemiológico da hanseníase na Microrregião de Tucuruí, estado do Pará, Brasil, nos anos de 2010 a 2014. MATERIAIS E METODOS: Foram coletados dados sobre os seis municípios da Microrregião de Tucuruí, referentes ao período de 2010 a 2014, disponibilizados no Sistema de Informação de Agravos de Notificação, organizados em planilhas e analisados estatisticamente no software BioEstat v5.3. RESULTADOS: A Microrregião de Tucuruí está na segunda posição em notificações de casos no Estado, na terceira na Região Norte e na $17^{a}$ do Brasil; em 2010, registrou 95,53/100.000 habitantes e notificou 1.786 casos no período de 2010 a 2014 , sendo $61,5 \%$ do sexo masculino; $45,0 \%$ na faixa etária de 15 a 39 anos; $66,5 \%$ com ensino fundamental como grau de instrução; e predominou a forma multibacilar $(68,1 \%)$. Aproximadamente um terço $(30,1 \%)$ da população estudada apresentou algum grau de incapacidade no momento do diagnóstico. É válido ressaltar que 214 casos ocorreram em menores de 15 anos de idade no período estudado, representando 12,0\% do total. CONCLUSÃO: A Microrregião ainda permanece no perfil de hiperendemicidade, com elevado número de casos entre menores de 15 anos de idade, e/ou algum grau de incapacidade após a alta por cura, o que demonstra a necessidade de adoção de políticas de educação em saúde voltadas para a população em situação de risco, visando o diagnóstico precoce.
\end{abstract}

Palavras-chave: Hanseníase; Epidemiologia Descritiva; Perfil de Saúde; Mycobacterium leprae.

\begin{abstract}
OBJECTIVE: To verify the epidemiological profile of leprosy in Tucuruí Microregion, Pará State, Brazil, from 2010 to 2014. MATERIALS AND METHODS: Data were collected on the six municipalities of Tucuruí Microregion from 2010 to 2014, available in the Brazilian Information System for Notifiable Diseases organized in spreadsheets and analyzed statistically in the BioEstat v5.3 software. RESULTS: Tucuruí Microregion is in the second position in case

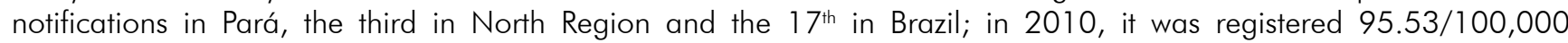
inhabitants and reported 1,786 cases from 2010 to 2014 , which $61.5 \%$ were male; $45.0 \%$ in the age group of 15 to 39 years old; $66.5 \%$ with elementary education as schooling status; and the multibacillary form predominated (68.1\%). Approximately one-third (30.1\%) of the study population presented some degree of disability at the time of diagnosis. It is important to note that 214 cases occured in children under 15 years old in the period studied, representing $12 \%$ of total. CONCLUSION: The Microregion still remains in the hyperendemicity profile with a high number of cases among children under 15 years old and/or some degree of disability after hospital discharge demonstrating the need to adopt health education policies for people at risk situation aiming early diagnosis.
\end{abstract}

Keywords: Leprosy; Descriptive Epidemiology; Health Profile; Mycobacterium leprae. 


\section{INTRODUÇÃO}

A hanseníase, ou mal de Hansen, é uma doença crônica granulomatosa causada pelo Mycobacterium leprae, bacilo álcool-ácido resistente, intracelular, que afeta nervos periféricos; apresenta grande capacidade de infecção (alta infectividade), porém poucos indivíduos desenvolvem a doença (baixa patogenicidade)'. A hanseníase é uma questão de saúde pública de grande relevância, pelo seu poder de causar incapacidades, atingindo todas as idades, especialmente a população economicamente ativa, entre os 15 e 40 anos de idade, com casos em todo o território brasileirol,2. Apesar de ter sido declarada a busca pela eliminação em escala mundial como um problema de saúde pública até o ano de $2000^{3}$, - Brasil ainda não alcançou a meta de reduzir a carga de hanseníase para menos de 1 caso/10.000 habitantes.

De acordo com a Organização Mundial da Saúde $(\mathrm{OMS})^{4}$, foram notificados, em 2010, 37.610 casos no Brasil, ficando atrás apenas da Índia, que registrou 133.717 casos. Dos 40.474 casos novos nas Américas, 93\% foram notificados no Brasil ${ }^{3}$. Segundo - Ministério da Saúde, em 2014, foram detectados 31.064 casos novos no Brasil, correspondente a um coeficiente de detecção geral de 15,32/100.000 habitantes, considerado muito alto. As Regiões Norte e Centro-Oeste apresentaram os maiores coeficientes de detecção geral de casos novos de hanseníase, com mais de 30 casos novos/100.000 habitantes 5 . Segundo Barreto et al. ${ }^{6}$, mais de 80.000 casos de hanseníase foram diagnosticados no período de 1995 a 2015 no Pará, e, até o momento deste estudo, a doença permanecia como um grave problema de saúde pública no Estado.

A OMS toma como modelo dois indicadores para avaliação do controle da infecção, sendo o primeiro o diagnóstico de novos casos em indivíduos na faixa etária abaixo de 15 anos, e o segundo, as notificações com grau 2 de incapacidade física ${ }^{7}$.

A partir da implantação do Sistema de Informação de Agravos de Notificação (Sinan) pelo Ministério da Saúde, tornaram-se viáveis explorações detalhadas de dados das mais variadas doenças em escalas geográficas, entre elas a hanseníase ${ }^{8}$. De acordo com a OMS', a meta mundial era diminuir em 35\% a taxa de detecção de novos casos com grau 2 de incapacidade a cada 100.000 habitantes, até o final de 2015, em comparação com os números de 2010. E a nova Estratégia Global para Hanseníase 2016-2020 tem o propósito de promover a detecção precoce da hanseníase e o tratamento imediato, para evitar a incapacidade e reduzir a transmissão da infecção na comunidade ${ }^{3}$.

○ conhecimento da epidemiologia local é importante para aumentar a detecção precoce de casos novos, sendo necessária sua utilização pelos municípios do Pará, para que o Estado possa finalmente alcançar as metas de controle da hanseníase. Entretanto, não foram encontrados, na literatura científica, levantamentos epidemiológicos da hanseníase na Microrregião de Tucuruí, o que, juntamente com os dados já encontrados na literatura sobre a situação do Pará em relação à hanseníase, especialmente a ausência de diagnóstico da doença ${ }^{10}$, pode justificar o presente estudo.

Diante do exposto, este estudo objetivou analisar o perfil epidemiológico da hanseníase na Microrregião de Tucuruí, estado do Pará, Brasil, no período de 2010 a 2014.

\section{MATERIAIS E MÉTODOS}

Trata-se de um estudo quantitativo epidemiológico, descritivo, de corte transversal, que teve como área de pesquisa a Microrregião de Tucuruí, cuja coleta de dados foi realizada em 2015 .

Segundo o plano de Desenvolvimento Regional Sustentável do Lago de Tucuruín ${ }^{11}$, essa Microrregião é formada pelas cidades de Breu Branco, Itupiranga, Jacundá, Nova Ipixuna, Novo Repartimento e Tucuruí, sendo esse último o polo da Microrregião, com uma distância aproximada de $400 \mathrm{~km}$ de Belém, a capital do Estado. Tucuruí é um dos 144 municípios do Pará e possuía a maior população da região, de acordo com o censo do Instituto Brasileiro de Geografia e Estatística (IBGE) realizado em 201012, com 105.451 habitantes; enquanto os outros município possuíam: Novo Repartimento, 69.267; Breu Branco, 59.651; Jacundá, 55.204; Itupiranga, 51.743; e Nova Ipixuna, 15.632 habitantes. Entretanto, em extensão territorial, se sobressai o município de Novo Repartimento, que possui $15.368,63 \mathrm{~km}^{2}$, o que representa $38,5 \%$ do total da Microrregião. Os menores são Tucuruí e Nova Ipixuna, com 1.600,32 e 2.086,17 km², respectivamente ${ }^{11}$.

Em relação ao Índice de Desenvolvimento Humano (IDH), apenas dois municípios superam a média regional, Tucuruí e Jacundá, que possuem os maiores IDH da região, 0,66 e 0,62, respectivamente. Contudo, apenas Tucuruí se enquadra na zona de médio desenvolvimento humano, enquanto os demais são de médio-baixo desenvolvimento. Novo Repartimento $(0,53)$ e Itupiranga $(0,58)$ têm os menores IDH da região ${ }^{12}$.

Todos os dados foram extraídos do Sinan ${ }^{13}$, tendo sido incluídos os casos de hanseníase de indivíduos infectados nas mais diversas localidades, porém, notificados nosmunicípios da Microrregião de Tucuruí, nos anos de 2010 a 2014. Os dados populacionais, paracálculo da taxa anual de detecção, foram obtidos a partir do $\mathrm{IBGE}^{12}$.

Foram destacadas as variáveis sexo, idade, escolaridade, raça/cor, forma clínica, classificação operacional, avaliação do grau de incapacidade durante o diagnóstico e esquema terapêutico para o tratamento ${ }^{14}$.

Para a análise estatística, os dados coletados foram organizados, filtrados e tabelados em planilhas no programa Microsoft Excel 2010. Foi feita a estatística 
descritiva utilizando-se o programa BioEstat v5.3. Para analisar as diferenças entre as frequências de algumas variáveis, foram usados o teste $G$ (aderência e independência) e 0 teste do qui-quadrado, assumindo-se o nível de significância de 5\% $(p<0,05)$.

O trabalho respeitou os preceitos da Resolução $\mathrm{n}^{\circ}$ da pesquisa 466/12 do Conselho Nacional de Saúde, que trata de pesquisa com seres humanos, sendo que os dados foram obtidos em bancos de dados fidedignos e de livre acesso, justificando-se, assim, a ausência do parecer de um Comitê de Ética em Pesquisa.

\section{RESULTADOS}

Entre 2010 e 2014, houve 1.786 casos notificados de hanseníase na Microrregião de Tucuruí, resultando em uma média de aproximadamente 358 casos por ano (Tabela 1).

Comparando-se os anos de 2010 e 2014, houve uma diminuição na taxa de detecção da hanseníase, tanto na Microrregião, como no Pará e no Brasil. No entanto, essa diminuição não foi significativa ( $\mathrm{p}<0,05)$. Apesar do declínio, o número ainda é alarmante quando comparado com as taxas do Pará e do Brasil, conforme mostra a figura 1 .

Tabela 1 - Casos de hanseníase na Microrregião de Tucuruí, estado do Pará, Brasil, de 2010 a 2014

\begin{tabular}{|c|c|c|c|c|c|c|}
\hline Município de notificação & 2010 & 2011 & 2012 & 2013 & 2014 & Total \\
\hline Breu Branco & 38 & 38 & 48 & 39 & 24 & 187 \\
\hline Itupiranga & 40 & 32 & 77 & 64 & 58 & 271 \\
\hline Jacundá & 85 & 149 & 117 & 52 & 72 & 475 \\
\hline Nova Ipixuna & 9 & 18 & 15 & 9 & 8 & 59 \\
\hline Novo Repartimento & 68 & 66 & 83 & 80 & 87 & 384 \\
\hline Tucuruí & 101 & 72 & 100 & 92 & 45 & 410 \\
\hline Total & 341 & 375 & 440 & 336 & 294 & 1.786 \\
\hline
\end{tabular}

O valor de $\mathrm{P}$ para todos os municípios foi $\mathrm{p}>0,05$.

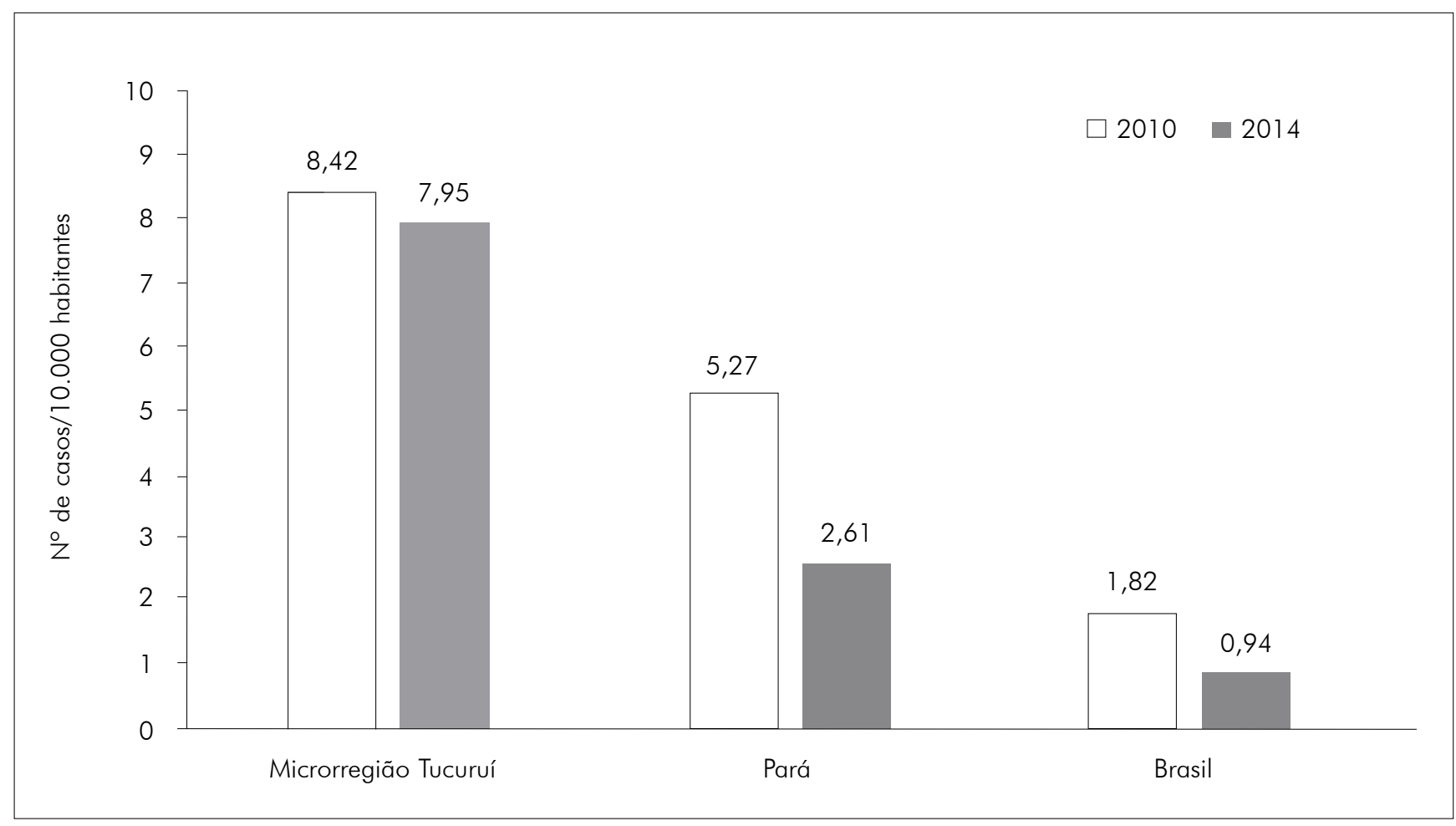

Teste $G, p=0,7444$.

Fonte: Sinan, 2015

Figura 1 - Taxa de detecção geral da hanseníase por 10.000 habitantes na Microrregião de Tucuruí, no estado do Pará e no Brasil, em 2010 e 2014 
Na Microrregião, o número de casos em menores de 15 anos de idade correspondeu a 12,0\% do total no período. A faixa etária predominante foi a de 15 a 39 anos $(45,0 \%)$, tendo sido observada pouca alteração entre os municípios, principalmente quanto aos menores de 15 anos (desvio padrão - DP $=1,33$ e coeficiente de variação - $C V=11,4 \%)$. A raça/cor parda foi a mais notificada $(61,1 \%)$, seguindo-se a preta $(21,1 \%)$ e a branca $(16,8 \%)$ (Tabela 2$)$.

$\mathrm{Na}$ análise das medidas de tendência central e dispersão, observou-se que a proporção média de indivíduos do sexo masculino foi de $61,5 \%$ dos casos (Tabela 2). Houve pouca variação entre os municípios (DP $=3,94$ e $C V=6,34 \%$ ), com a proporção de homens maior que a de mulheres em todos os anos.

A escolaridade predominante das pessoas afetadas em todos os municípios da Microrregião foi o ensino fundamental $(66,5 \%)$, vindo em seguida os analfabetos (14,2\%), com diferença $p<0,0001$. $\bigcirc$ grau de escolaridade de 9,2\% dos casos encontrava-se em ignorado/branco/não se aplica (Tabela 2).

A forma clínica predominante na área do estudo foi a dimorfa (53,5\%; 955/1.786), com mais de cinco lesões, multibacilar $(68,1 \%)$. A indeterminada, que se constitui na forma inicial da doença, foi a segunda em prevalência $(20,7 \% ; 369 / 1.786)$ (Figura 2).

Tabela 2 - Perfil dos pacientes na Microrregião de Tucuruí, estado do Pará, Brasil, de 2010 a 2014

\begin{tabular}{|c|c|c|c|c|c|c|c|c|c|c|c|c|c|c|}
\hline \multirow{2}{*}{ Variáveis de perfil } & \multicolumn{2}{|c|}{ Breu Branco } & \multicolumn{2}{|c|}{ Itupiranga } & \multicolumn{2}{|c|}{ Jacundá } & \multicolumn{2}{|c|}{ Nova Ipixuna } & \multicolumn{2}{|c|}{$\begin{array}{c}\text { Novo } \\
\text { Repartimento }\end{array}$} & \multicolumn{2}{|c|}{ Tucuruí } & \multicolumn{2}{|c|}{ Total } \\
\hline & $\mathrm{N}$ & $\%$ & $\mathrm{~N}$ & $\%$ & $\mathrm{~N}$ & $\%$ & $\mathrm{~N}$ & $\%$ & $\mathrm{~N}$ & $\%$ & $\mathrm{~N}$ & $\%$ & $\mathrm{~N}$ & $\%$ \\
\hline \multicolumn{15}{|l|}{ Idade (anos) } \\
\hline$<15$ & 20 & 10,7 & 34 & 12,6 & 51 & 10,8 & 6 & 10,2 & 52 & 13,5 & 51 & 12,4 & 214 & 12,0 \\
\hline $15-39$ & 89 & 47,6 & 109 & 40,2 & 211 & 44,4 & 24 & 40,7 & 171 & 44,5 & 199 & 48,5 & 803 & 45,0 \\
\hline $40-59$ & 54 & 28,9 & 80 & 29,5 & 153 & 32,2 & 21 & 35,6 & 117 & 30,5 & 111 & 27,1 & 536 & 30,0 \\
\hline$\geq 60$ & 24 & 12,8 & 48 & 17,7 & 60 & 12,6 & 8 & 13,5 & 44 & 11,5 & 49 & 12,0 & 233 & 13,0 \\
\hline Subtotal & 187 & 100,0 & 271 & 100,0 & 475 & 100,0 & 59 & 100,0 & 384 & 100,0 & 410 & 100,0 & 1.786 & 100,0 \\
\hline $\mathrm{p}$-valor & \multicolumn{2}{|c|}{$<0,0001$} & \multicolumn{2}{|c|}{$<0,0001$} & \multicolumn{2}{|c|}{$<0,0001$} & \multicolumn{2}{|c|}{0,0008} & \multicolumn{2}{|c|}{$<0,0001$} & \multicolumn{2}{|c|}{$<0,0001$} & \multicolumn{2}{|c|}{$<0,0001$} \\
\hline \multicolumn{15}{|l|}{ Sexo } \\
\hline Masculino & 110 & 58,8 & 164 & 60,5 & 278 & 58,5 & 40 & 67,8 & 255 & 66,4 & 251 & 61,2 & 1.098 & 61,5 \\
\hline Feminino & 77 & 41,2 & 107 & 39,5 & 197 & 41,5 & 19 & 32,2 & 129 & 33,6 & 159 & 38,8 & 688 & 38,5 \\
\hline Subtotal & 187 & 100,0 & 271 & 100,0 & 475 & 100,0 & 59 & 100,0 & 384 & 100,0 & 410 & 100,0 & 1.786 & 100,0 \\
\hline p-valor & \multicolumn{2}{|c|}{0,0193} & \multicolumn{2}{|c|}{0,0007} & \multicolumn{2}{|c|}{0,0002} & \multicolumn{2}{|c|}{0,0092} & \multicolumn{2}{|c|}{$<0,0001$} & \multicolumn{2}{|c|}{$<0,0001$} & \multicolumn{2}{|c|}{$<0,0001$} \\
\hline \multicolumn{15}{|l|}{ Raça/cor } \\
\hline Branca & 36 & 19,2 & 40 & 14,8 & 73 & 15,4 & 11 & 18,7 & 66 & 17,2 & 74 & 18,1 & 300 & 16,8 \\
\hline Preta & 40 & 21,4 & 50 & 18,4 & 105 & 22,1 & 17 & 28,8 & 75 & 19,5 & 89 & 21,7 & 376 & 21,1 \\
\hline Amarela & 1 & 0,5 & - & - & 1 & 0,2 & - & - & 5 & 1,3 & 3 & 0,7 & 10 & 0,6 \\
\hline Parda & 108 & 57,8 & 181 & 66,8 & 293 & 61,7 & 31 & 52,5 & 237 & 61,7 & 242 & 59,0 & 1.092 & 61,1 \\
\hline Indígena & - & - & - & - & 2 & 0,4 & - & - & - & - & 2 & 0,5 & 4 & 0,2 \\
\hline $\begin{array}{l}\text { lgnorado/ } \\
\text { branco }\end{array}$ & 2 & 1,1 & - & - & 1 & 0,2 & - & - & 1 & 0,3 & - & - & 4 & 0,2 \\
\hline Subtotal & 187 & 100,0 & 271 & 100,0 & 475 & 100,0 & 59 & 100,0 & 384 & 100,0 & 410 & 100,0 & 1.786 & 100,0 \\
\hline p-valor & \multicolumn{2}{|c|}{$<0,0001$} & $<0$ & 0001 & $<0$ & 0001 & & 0001 & $<0$, & 0001 & $<0$, & 0001 & $<0$, & 0001 \\
\hline Escolaridade & & & & & & & & & & & & & & \\
\hline Analfabeto & 27 & 14,4 & 51 & 18,8 & 75 & 15,8 & 12 & 20,3 & 54 & 14,1 & 35 & 8,5 & 254 & 14,2 \\
\hline Fundamental & 126 & 67,4 & 182 & 67,2 & 363 & 76,4 & 28 & 47,5 & 206 & 53,6 & 282 & 68,8 & 1.187 & 66,5 \\
\hline Médio & 29 & 15,5 & 26 & 9,6 & 29 & 6,1 & 3 & 5,1 & 25 & 6,5 & 57 & 14,0 & 169 & 9,4 \\
\hline Superior & 1 & 0,5 & 2 & 0,7 & 2 & 0,4 & 1 & 1,7 & 3 & 0,8 & 3 & 0,7 & 12 & 0,7 \\
\hline $\begin{array}{l}\text { Ignorado/ } \\
\text { branco/não } \\
\text { se aplica }\end{array}$ & 4 & 2,2 & 10 & 3,7 & 6 & 1,3 & 15 & 25,4 & 96 & 25,0 & 33 & 8,0 & 164 & 9,2 \\
\hline Subtotal & 187 & 100,0 & 271 & 100,0 & 475 & 100,0 & 59 & 100,0 & 384 & 100,0 & 410 & 100,0 & 1.786 & 100,0 \\
\hline$p$-valor & $<0$ & 0001 & $<0$ & 0001 & $<0$ & 0001 & $<0$ & 0001 & $<0$, & 0001 & $<0$, & 001 & $<0$, & 0001 \\
\hline
\end{tabular}




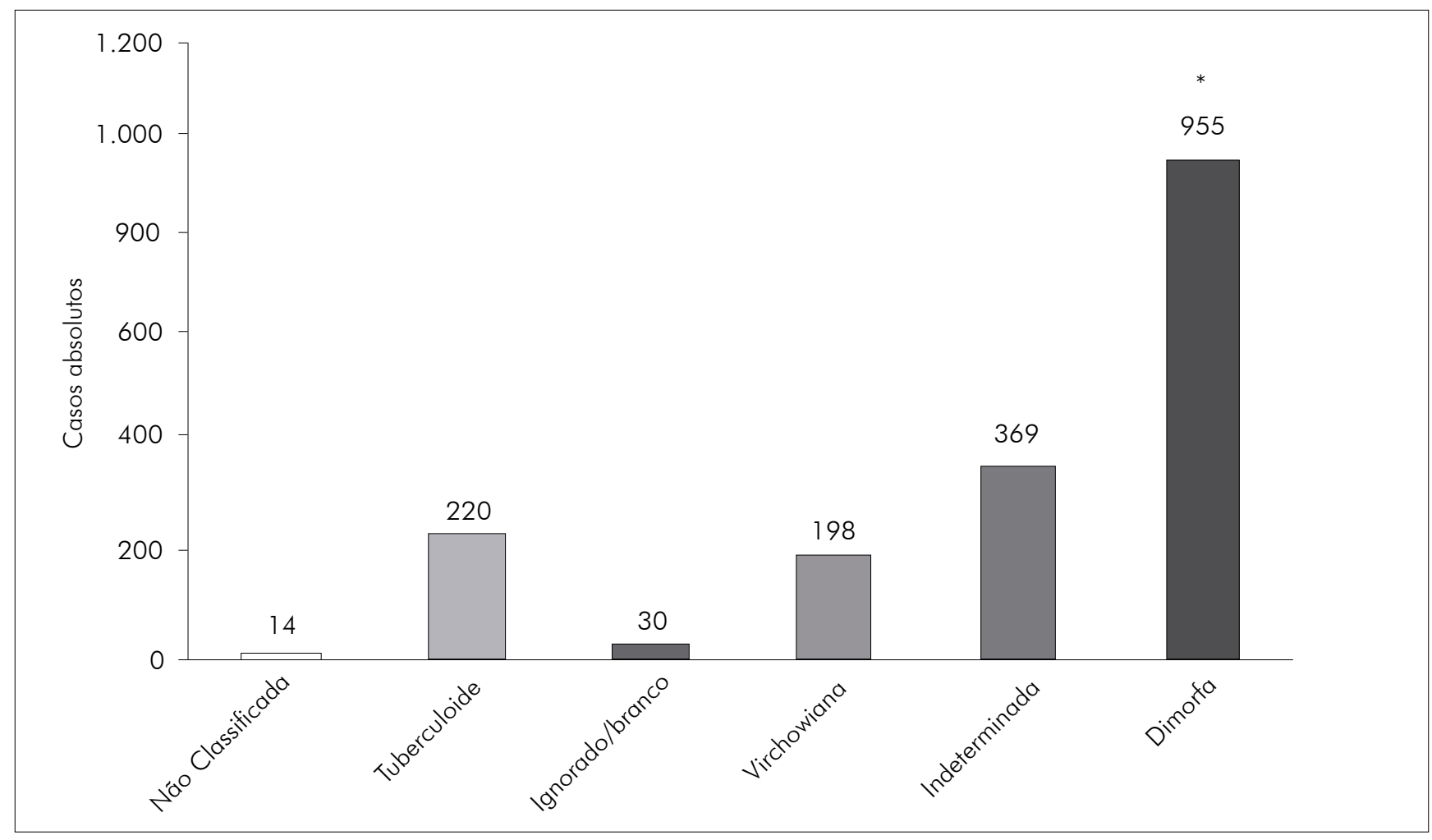

* Valor de $\mathrm{p}<0,0001$.

Fonte: Sinan, 2015

Figura 2 - Forma clínica de hanseníase na Microrregião de Tucuruí, estado do Pará, Brasil, de 2010 a 2014

Quanto ao grau de incapacidades, aproximadamente um terço $(30,1 \%)$ da população estudada apresentou algum grau de incapacidade no momento do diagnóstico, tendo sido a maior parte de grau 1 (426 casos), o que é mostrado na tabela 3.

Na análise sociodemográfica, 31,7\% dos pacientes com 35 anos ou mais apresentaram algum grau de incapacidade, e $21,7 \%$ dos pacientes abaixo de 35 anos apresentaram algum grau de incapacidade, sendo os mesmos com baixo nível de escolaridade, havendo a mesma proporção entre homens e mulheres.
No entanto, na avaliação do grau de incapacidade de cura (pós-poliquimioterapia), aproximadamente metade dos casos $(47,0 \%)$ foram preenchidos como ignorado/branco, não permitindo assim uma análise precisa de uma tendência ao longo do período estudado. $O$ esquema terapêutico PQT/MB 12 doses foi o mais aplicado $(67,4 \%)$. A cura foi alcançada em 933 casos $(52,2 \%)$, e houve $57(3,1 \%)$ abandonos de tratamento; porém, $681(38,1 \%)$ fichas foram enviadas para a base do Sinan sem o preenchimento da evolução dos casos.

Tabela 3 - Grau de incapacidade notificada por município na Microrregião de Tucuruí, estado do Pará, Brasil, de 2010 a 2014

\begin{tabular}{|c|c|c|c|c|c|c|c|c|c|c|c|c|}
\hline \multirow{3}{*}{$\begin{array}{l}\text { Município de } \\
\text { notificaçãoo }\end{array}$} & \multirow{2}{*}{\multicolumn{2}{|c|}{$\begin{array}{c}\text { Ignorado/ } \\
\text { branco }\end{array}$}} & \multicolumn{8}{|c|}{ Grau de incapacidade classificado (número de casos) } & \multirow{2}{*}{\multicolumn{2}{|c|}{ Total }} \\
\hline & & & \multicolumn{2}{|c|}{ Grau 0} & \multicolumn{2}{|c|}{ Grau 1} & \multicolumn{2}{|c|}{ Grau 2} & \multicolumn{2}{|c|}{ Não avaliado } & & \\
\hline & $\mathrm{N}$ & $\%$ & $\mathrm{~N}$ & $\%$ & $\mathrm{~N}$ & $\%$ & $\mathrm{~N}$ & $\%$ & $\mathrm{~N}$ & $\%$ & $\mathrm{~N}$ & $\%$ \\
\hline Breu Branco & 2 & 1,1 & 90 & 48,1 & 58 & 31,0 & 36 & 19,3 & 1 & 0,5 & 187 & 100,0 \\
\hline Itupiranga & 2 & 0,7 & 197 & 72,7 & 68 & 25,1 & 3 & 1,1 & 1 & 0,4 & 271 & 100,0 \\
\hline Jacundá & - & - & 380 & 80,0 & 81 & 17,1 & 12 & 2,5 & 2 & 0,4 & 475 & 100,0 \\
\hline Nova Ipixuna & 3 & 5,1 & 31 & 52,5 & 16 & 27,1 & 7 & 11,9 & 2 & 3,4 & 59 & 100,0 \\
\hline Novo Repartimento & 9 & 2,3 & 247 & 64,3 & 102 & 26,6 & 19 & 5,0 & 7 & 1,8 & 384 & 100,0 \\
\hline Tucuruí & 2 & 0,5 & 263 & 64,2 & 101 & 24,6 & 35 & 8,5 & 9 & 2,2 & 410 & 100,0 \\
\hline Total & 18 & 1,0 & 1.208 & 67,7 & 426 & 23,8 & 112 & 6,3 & 22 & 1,2 & 1.786 & 100,0 \\
\hline
\end{tabular}




\section{DISCUSSÃO}

Na Microrregião estudada, a taxa de detecção de hanseníase foi maior do que no estado do Pará e no país, alcançando 7,95 no ano de 2014. De acordo com o padrão do Ministério da Saúde ${ }^{14}$, essa taxa é considerada hiperendêmica, por apresentar, em todos os anos do estudo (2010 a 2014), coeficientes de detecção maiores que 4 casos novos/10.000 habitantes. Esse fato é ainda mais grave, pois Barreto et al. ${ }^{6}$ mostraram haver uma alta taxa de prevalência oculta de hanseníase e de infecção subclínica pelo $M$. leprae no Pará. De acordo com Blok et al. ${ }^{15}$, até 2020 haverá uma tendência decrescente; todavia, em lugares de alta endemicidade, como no Pará, a meta de eliminação para menos de 1 caso/10.000 habitantes ainda não será alcançada. Adicionalmente, modelos matemáticos mais recentes preveem que, mantidas as condições atuais, as Regiões Norte, Nordeste e CentroOeste do Brasil não deverão alcançar a eliminação da hanseníase como problema de saúde pública em menos de 44 a 45 anos $^{16}$.

○ número de novos casos de hanseníase encontrados em uma área pode sofrer influência de ações de educação e saúde, de controle da doença, bem como da competência dos profissionais de saúde para o diagnóstico exato e precoce ${ }^{9,17}$. Municípios com poucos habitantes e um número de casos novos baixos resultam em altos coeficientes, - que pode mascarar a realidade epidemiológica do município ${ }^{18,19}$. Essa é a realidade do município de Nova Ipixuna, cuja população pequena apresentou coeficiente de detecção de 12,11/10.000 habitantes em 2011, tornando-o assim homogêneo em relação aos demais municípios da Microrregião. Apesar da diferença de notificações entre os municípios, todos permaneneceram no parâmetro de hiperendemicidade nos anos do estudo, sinalizando a necessidade de medidas de controle na região. Além disso, a hanseníase indeterminada foi a segunda mais prevalente $(20,7 \%)$ neste estudo; ela se constitui na forma inicial da doença e pode evolvir para cura espontânea ou para formas polarizadas ${ }^{20}$, merecendo atenção quanto ao seu controle.

Com relação aos dados de perfil nos municípios da Microrregião, a faixa etária entre 15 e 59 anos foi a prevalente, o que permite pressupor que a faixa economicamente ativa é a mais afetada pela infecção por $M$. leprae. Miranzi et al. ${ }^{21}$ afirmaram que pode haver prejuízos para a economia dessa área, uma vez que essa classe pode desenvolver múltiplas incapacidades físicas, como lesões, reações hansênicas e, por fim, a exclusão do mercado de trabalho, sendo isso um grande prejuízo social. Outros estudos corroboram esses achados, como os realizados em Divinópolis, estado de Minas Gerais ${ }^{17}$ e no estado da Paraíba ${ }^{22}$.

Em 2010 e 2011, os casos em menores de 15 anos de idade corresponderam a 7,1\% do total de casos novos no Brasil ${ }^{2,23}$. Na Microrregião de
Tucuruí, a média de casos em menores de 15 anos foi de 214 casos, representando $12,0 \%$ do total de casos notificados no período estudado, sendo observado um coeficiente de variação de 15,3\% entre os anos; ressalta-se que houve aumento de casos entre o primeiro e o último ano da pesquisa. Segundo informações do Sinan ${ }^{24}$, em 2010, a quantidade de casos de hanseníase, no Brasil, foi de 34.894 casos novos, sendo $2.461(7,1 \%)$ em menores de 15 anos, e o coeficiente geral de detecção era de 18,2 para cada 100.000 habitantes. A existência de casos em menores de 15 anos indica que há circuitos de transmissão ativos, o que sugere um contágio nos primeiros anos de vida - uma das características de regiões com intensa transmissão da doença - e significa que medidas precisam ser tomadas ${ }^{22}$.

Dessa forma, é necessário manter a vigilância sobre a população mais jovem, porque o aumento dos casos e a situação de adoecimento na faixa etária infantojuvenil mostraram que há transmissão recente e ativa que precisa ser controlada, sendo o aumento de casos em menores de 15 anos um indicador de aumento de portadores bacilíferos. Esses, sem tratamento, poderão aumentar o índice de infecção, pois, de outra forma, se há diminuição da transmissão da doença, o número de crianças afetadas também diminuiráa ${ }^{2,9}$; essa diminuição não está ocorrendo na Microrregião pesquisada. Com isso, espera-se que a vigilância epidemiológica possa promover a busca ativa em menores de 15 anos de idade, no sentido de detectar a doença precocemente, para diminuir os riscos de se contrair hanseníase ${ }^{25}$.

Para Brito et al. ${ }^{22}$, a variável raça/cor é pouco considerada, e, em estudos onde é avaliada, essa variável está mais relacionada com a região de estudo do que com a doença propriamente dita, uma vez que, no Brasil, existe muita miscigenação, e a maioria da população é de cor parda. Assim, na Microrregião de Tucuruí, foi encontrada a maior prevalência de doentes de raça/cor parda $(61,1 \%)$, devido à grande miscigenação.

Quanto ao gênero, observou-se a predominância de hanseníase no sexo masculino, com média de 220 casos/ano (61,5\%; p $<0,0001)$, corroborando outros achados ${ }^{17,22}$. De acordo com a OMS ${ }^{26}$, embora a hanseníase comprometa tanto homens quanto mulheres, na maior parte do mundo, o sexo masculino é o mais afetado, com proporção de 2:1. Deve ser salientado que, particularmente na África, há ocorrência igual de hanseníase em ambos os sexos, e algumas vezes prevalece no sexo feminino ${ }^{22}$.

Os achados sobre a escolaridade neste estudo apenas ratificam o que já se encontra na literatura, em que se observa uma predominância de hanseníase em indivíduos com baixos níveis de escolaridade ${ }^{22,27}$, reforçando a tese de que fatores sociais têm forte relação com a ocorrência dessa doença no Brasil ${ }^{28}$. Dessa forma, Amaral e Lana $^{25}$ sugeriram que, no momento do planejamento das atividades de educação 
e saúde, as instituições levassemem consideração o nível de conhecimento da população, com o objetivo de garantirem o entendimento.

Em relação à forma clínica e ao grau de incapacidades, a Microrregião de Tucuruí indica que houve uma possível falha no diagnóstico precoce da hanseníase, pois $68,1 \%$ dos casos apresentavam mais de cinco lesões (multibacilar), com mais da metade do total classificada como dimorfa; além disso, a cada três casos, pelo menos um apresentava algum grau de incapacidade. Segundo Costa e Patrus ${ }^{29}$, a presença de incapacidades no momento do diagnóstico pode indicar atraso, tendo em vista que tardiamente a hanseníase causa grandes incapacidades nos pacientes, sinalizando a necessidade de um melhor controle.

Amaral e Lana ${ }^{25}$ ressaltaram que as incapacidades físicas e deformidades são os principais problemas da hanseníase, pois, como já mencionado anteriormente, essa doença atinge principalmente a faixa economicamente ativa, logo as incapacidades podem afetar as atividades dos pacientes, tornando-os incapazes de fazerem parte do setor econômico, o que leva ao desenvolvimento de problemas sociais e psicológicos, diminuindo drasticamente a qualidade de vida $^{30}$. Diante disso, Pimentel et al. ${ }^{31}$ explicaram que, durante o diagnóstico, é imprescindível a realização do exame dermatoneurológico, para verificação do grau de incapacidade.

$\bigcirc$ percentual de incapacidades na Microrregião é superior ao encontrado por Ribeiro Júnior et al. ${ }^{32}$, em um estudo realizado no norte de Minas Gerais, porém, inferior ao encontrado por Sobrinho et al. ${ }^{33}$, em uma região do estado do Paraná. Vale ressaltar que é perceptível uma discrepância em relação ao grau de incapacidade de cura entre os municípios da mesma Microrregião, o que pode gerar hipótese de falhas na busca ativa da hanseníase em determinados municípios.

De acordo com Lana et al. ${ }^{34}$, as consequências negativas da hanseníase na vida do indivíduo podem ser agravadas se a doença ocorrer na infância, e isso torna preocupante o quadro encontrado na Microrregião quanto à incapacidade em menores de 15 anos de idade.

A literatura aponta uma relação entre o atraso do diagnóstico da hanseníase por mais de um ano e a classificação da doença como multibacilar, com presença de incapacidade física ${ }^{35}$. $\bigcirc$ objetivo maior das ações de controle da doença é o diagnóstico precoce, sendo esperado um predomínio da forma clínica indeterminada, pois, segundo Gomes et al. ${ }^{36}$, em algumas áreas onde a hanseníase foi considerada eliminada, houve aumento no grau de incapacidades. Dessa forma, é indispensável realizar atividades de controle e pesquisa sobre a hanseníase, ainda que sejam em áreas nas quais a doença foi oficialmente erradicada ${ }^{20,36}$.
Além disso, a avaliação e a prevenção das incapacidades físicas devem acontecer em conjunto com a poliquimioterapia, iniciada precocemente para interromper a cadeia de transmissão do M. leprae, pois isso, juntamente com outras intervenções específicas, aumenta a possibilidade de controle da hanseníase 25,33

Entre os trabalhos primordiais do Ministério da Saúde, está o Programa Nacional de Controle da Hanseníase. Atualmente, a política nacional de controle da doença trabalha para que a hanseníase deixe de ser um problema de saúde pública no Brasil, e, para isso, anseia-se diminuir a prevalência para 1 ou menos de 1 caso/10.000 habitantes ${ }^{25}$. Entretanto, em um estudo sobre a capacitação da equipe de enfermagem ${ }^{33}$, foi ressaltado que é imprescindível que o diagnóstico precoce seja efetivo, observando-se principalmente o diagnóstico de casos em menores de 15 anos de idade, para diminuição das incapacidades físicas. Mas, para isso, é necessária a capacitação dos profissionais de saúde.

Entre outras atribuições da equipe de enfermagem, está o preenchimento das fichas de notificação. Neste estudo, houve um grande problema, tendo em vista que $38,1 \%$ das fichas não foram preenchidas em relação à evolução dos casos, figurando apenas como ignorado ou em branco, o que dificultou traçar um perfil de cura. Para Brito et al. ${ }^{22}$, o preenchimento inadequado pelos profissionais ou a perda de dados durante a alimentação do sistema é uma das arestas enfrentadas em se trabalhar com sistemas de informações. Os autores do referido estudo evidenciaram a necessidade de treinamento profissional.

Diante disso, Sobrinho et al. ${ }^{33}$ afirmaram que se faz necessário um trabalho de educação permanente junto aos municípios, promovendo-se discussões, com a colaboração de profissionais capacitados para diagnosticar, avaliar e classificar o grau de incapacidade física dos pacientes, como atividades de rotina.

\section{CONCLUSÃO}

No presente estudo, as notificações de casos de hanseníase, ocorridas nos anos de 2010 a 2014, não tiveram mudanças significativas, e a Microrregião pesquisada permanece no parâmetro de hiperendemicidade. A hanseníase afeta, principalmente, pessoas na faixa etária economicamente ativa, de baixa escolaridade, tendo sido a forma multibacilar a mais encontrada. Também se evidenciaram altos índices de casos em menores de 15 anos de idade, e muitos casos com algum grau de incapacidade, o que indica o descontrole da doença na região. Assim, a hanseníase ainda é um problema de saúde pública na Microrregião de Tucuruí. 
Os dados revelaram a importância das ações de vigilância em saúde de cada município para a diminuição e o controle dessa doença - até mesmo em Nova Ipixuna, onde houve o menor número de casos relatados - bem como medidas de capacitação dos profissionais quanto ao diagnóstico, a busca ativa e a notificação da doença.
Medidas de busca ativa precisam ser realizadas na região, visando ao diagnóstico precoce e à diminuição das incapacidades provocadas pela doença.

\section{CONFLITOS DE INTERESSE}

Os autores declaram não haver conflitos de interesses neste estudo.

\section{REFERÊNCIAS}

1 Ministério da Saúde (BR). Secretaria de Vigilância em Saúde. Departamento de Vigilância Epidemiológica. Doenças infecciosas e parasitárias: guia de bolso. 8. ed. rev. Brasília: Ministério da Saúde; 2010. 32 p. (Série B. Textos básicos de saúde).

2 Ministério da Saúde (BR). Secretaria de Atenção a Saúde. Departamento de Atenção Básica. Vigilância em Saúde. Brasília: Ministério da Saúde; 2007. 70 p. (Cadernos de atenção básica; no. 21).

3 World Health Organization. Global leprosy strategy: accelerating towards a leprosy-free world [Internet]. Geneva: WHO; 2016 [cited 2016 Nov 10]. Available from: http://www.wpro.who.int/leprosy/ documents/globalleprosystrategy2016-2020.pdf.

World Health Organization. Global leprosy situation, 2010. Wkly Epidemiol Rec [Internet]. 2010 Aug [cited 2015 Feb 5];85(35):338-9. Available from: http://www.who.int/wer/2010/wer8535.pdf?ua =1.

5 Ministério da Saúde (BR). Secretaria de Vigilância em Saúde. Indicadores de Vigilância em Saúde descritos segundo a variável raça/cor, Brasil. Bol Epidemiol [Internet]. 2017 fev [citado 2017 jul 30];48(4):1-35. Disponível em: http:// portalarquivos.saude.gov.br/images/pdf/2017/ fevereiro/17/Indicadores-de-Vigilancia-em-Saudedescritos-segundo-ra--a-cor.pdf.

6 Barreto JG, Bisanzio D, Frade MAC, Moraes TMP, Gobbo AR, Guimarães LS, et al. Spatial epidemiology and serologic cohorts increase the early detection of leprosy. BMC Infect Dis. 2015 Nov; 15:527.

7 Ministério da Saúde (BR). OMS adota indicadores do Brasil para controlar hanseníase [Internet]. Brasília: Ministério da Saúde; 2009 [citado 2015 fev 10]. Disponível em: http://portal. saude.gov.br/portal/aplicacoes/noticias/default. $\mathrm{cfm}$ ? pg $=$ dspDetalheNoticia\&id_area $=124 \& \mathrm{CO}_{-}$ NOTICIA $=10441$.

8 Magalhães MCC, Rojas LI. Diferenciação territorial da hanseníase no Brasil. Rev Epidemiol Serv Saude. 2007 jun; 16(2):76-84.
9 Organização Pan-Americana da Saúde. Estratégia global aprimorada para redução adicional da carga da hanseníase: período do plano: 2011 2015. Brasília: OPAS; 2010.

10 Salgado CG, Barreto JG, Silva MB, Frade MAC, Spencer JS. What do we actually know about leprosy worldwide? Lancet Infect Dis. 2016 Jul; 16(7):778.

11 Secretaria de Estado e Integração Regional (Pará). Plano de desenvolvimento regional sustentável do Lago de Tucuruí [Internet]. Belém: Secretaria de Estado e Integração Regional; 2013 [citado 2015 fev 10]. 153 p. Disponível em: http://www.mi.gov. $\mathrm{br} / \mathrm{c} /$ document_library/get_file? uvid=0abeac82 0b58-40bf-a60e-c80828c0ec90\&groupld $=10157$.

12 Instituto Brasileiro de Geografia e Estatística. Censo 2010 [Internet]. Brasília: IBGE; 2010 [citado 2015 fev 5]. Disponível em: http://censo2010.ibge.gov. $\mathrm{br} /$.

13 Ministério da Saúde (BR). Departamento de Informática do SUS. Sistema de Informação de Agravos de Notificação - SINAN [Internet]. Brasília: Ministério da Saúde; 2015 [citado 2015 jan 20]. Disponível em: http://www.datasus.gov.br.

14 Brasil. Ministério da Saúde. Secretaria de Vigilância em Saúde. Portaria $\mathrm{n}^{\circ} 3.125$, de 7 de outubro de 2010. Aprova as diretrizes para vigilância, atenção e controle da hanseníase. Diário Oficial da União, Brasília (DF), 2010 out 15; Seção 1:55.

15 Blok DJ, De Vlas SJ, Richardus JH. Global elimination of leprosy by 2020: are we on track? Parasit Vec. 2015 Oct;8:548.

16 Smith RL. Proposing a compartmental model for leprosy and parameterizing using regional incidence in Brazil. PLoS Negl Trop Dis. 2016 Aug;10(8):e0004925.

17 Lanza FM, Cortez DN, Gontijo TL, Rodrigues JSJ. Perfil epidemiológico da hanseníase no município de Divinópolis, Minas Gerais. Rev Enferm UFSM. 2012 mai-ago;2(2):365-74.

18 Lana FCF, Carvalho APM, Davi RFL. Perfil epidemiológico da hanseníase na microrregião de Araçuaí e sua relação com ações de controle. Esc Anna Nery. 2011 jan-mar;15(1):62-7. 
19 Lastória JC, Putinatti SMA. Utilização de busca ativa de hanseníase: relato de uma experiência de abordagem na detecção de casos novos. Hansen Int. 2004 jan-jun;29(1):6-11.

20 Talhari S, Neves RG. Dermatologia tropical hanseníase. 3. ed. Manaus: Gráfica Tropical; 1997.

21 Miranzi SSC, Pereira LHM, Nunes AA. Perfil epidemiológico da hanseníase em um município brasileiro, no período de 2000 a 2006. Rev Soc Bras Med Trop. 2010 jan-fev;43(1):62-7.

22 Brito KKG, Araújo DAL, Uchôa REMN, Ferreira JDL, Soares MJDO, Lima JO. Epidemiologia da hanseníase em um estado do nordeste brasileiro. Rev Enferm UFPE. 2014 ago;8(8):2686-93.

23 Ministério da Saúde (BR). Secretaria de Vigilância em Saúde. Informe epidemiológico: hanseníase: Fortaleza-CE [Internet]. Brasília: Ministério da Saúde; 2013 [citado 2015 jan 20]. Disponível em: http://portalsaude.saude.gov.br/portalsaude/ arquivos/pdf/2013/Jul/23/2013_009_boletim_ hanseniase.pdf.

24 Ministério da Saúde (BR). Secretaria de Vigilância Epidemiológica. Programa Nacional de Hanseníase. Situação epidemiológica da hanseníase no Brasil - 2010 [Internet]. Brasília: Ministério da Saúde; 2011 [citado 2015 fev 15]. Disponível em: http://www.mpf.mp.br/atuacao-tematica/pfdc/ institucional/grupos-de-trabalho/saude/atuacao/ memorias/2011/apresentacao-sobre-hanseniasereuniao-de-21-e-22-novembro-2011.

25 Amaral EP, Lana FCF. Análise espacial da hanseníase na microrregião de Almenara, MG, Brasil. Rev Bras Enferm. 2008 nov;61(no. esp):701-7.

26 World Health Organization. Leprosy today [Internet]. Geneva: WHO; 2010 [cited 2015 Jul 13]. Available from: http://www.who.int/lep/transmission/ en/index $5 . h t m l$.

27 Macedo TDC, Ramos ARS. Características dos portadores de hanseníase por região brasileira no período de 2000 a 2011 . In: Anais da $5^{\circ}$ Jornada Científica da UNEMAT: $8^{\circ}$ Congresso de Iniciação Científica; $4^{\circ}$ Seminário de Extensão Universitária; $2^{\circ}$ Seminário PIBID; 2013 abr 22-24; Cárceres, Mato Grosso. Cárceres (MT): UNEMAT; 2013.
28 Richardus JH, Habbema JD. The impact of leprosy control on the transmission of M. leprae: is elimination being attained? Lepr Rev. 2007 Dec;78(4):330-7.

29 Costa IMC, Patrus AO. Incapacidades físicas em pacientes de hanseníase na faixa de zero a 14 anos, no Distrito Federal, no período de 1979 a 1989. An Bras Dermatol. 1992;67(5):245-9.

30 Duarte MTC, Ayres JA, Simonetti JP. Socioeconomic and demographic profile of leprosy carriers attended in nursing consultations. Rev Latino-Am Enferm. 2007 Sep-Oct; 15(Spec No.):774-9.

31 Pimentel MIF, Nery JAC, Borges E, Gonçalves $R R$, Sarno EN. $O$ exame neurológico inicial na hanseníase multibacilar: correlação entre a presença de nervos afetados com incapacidades presentes no diagnóstico e com a ocorrência de neurites francas. Anais Bras Dermatol. 2003 set-out; 78(5):561-8.

32 Ribeiro Júnior AF, Vieira MA, Caldeira AP. Perfil epidemiológico da hanseníase em uma cidade endêmica no Norte de Minas Gerais. Rev Bras Clin Med. 2012 jul-ago; 10(4):272-7.

33 Silva Sobrinho RA, Mathias TAF, Gomes EA, Lincoln PB. Avaliação do grau de incapacidade em hanseníase: uma estratégia para sensibilização e capacitação da equipe de enfermagem. Rev Latino-Am Enferm. 2007 nov-dez; 15(6):1 1 25-30.

34 Lana FCF, Amaral EP, Lanza FM, Lima PL, Carvalho ACN, Diniz LG. Hanseníase em menores de 15 anos no Vale do Jequitinhonha, Minas Gerais, Brasil. Rev Bras Enferm. 2007 nov-dez; 60(6):696-700.

35 Guerrero Ml, Muvdi S, León Cl. Retraso en el diagnóstico de lepra como factor pronóstico de discapacidad en una cohorte de pacientes en Colombia, 2000 - 2010. Rev Panam Salud Publica. 2013 feb;33(2): 137-43.

36 Gomes CCD, Pontes MAA, Gonçalves HS, Penna GO. Perfil clínico-epidemiológico dos pacientes diagnosticados com hanseníase em um centro de referência na região nordeste do Brasil. An Bras Dermatol. 2005 nov-dez;80 supl 3:S283-8. 